\title{
Optical filter based on a coupled bilayer photonic crystal
}

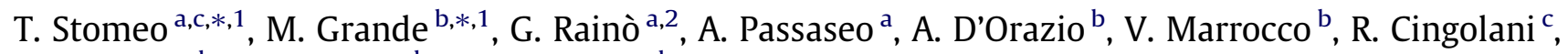 \\ A. Locatelli ${ }^{\mathrm{d}}$, D. Modotto $^{\mathrm{d}}$, C. De Angelis $^{\mathrm{d}}$, M. De Vittorio ${ }^{\mathrm{a}, \mathrm{c}}$ \\ a National Nanotechnology Laboratory (NNL), CNR-ISTITUTO DI NANOSCIENZE, Dip. Ingegneria dell'Innovazione, Università Del Salento, Via Arnesano, 73100 Lecce, Italy \\ ${ }^{\mathrm{b}}$ Dipartimento di Elettrotecnica ed Elettronica, Politecnico di Bari, Via Re David 200, 70125 Bari, Italy \\ 'Italian Institute of Technology (IIT), Center for Bio-Molecular Nanotechnology, Via Barsanti 1, Arnesano (Lecce) 73010, Italy \\ ${ }^{\mathrm{d}}$ Dipartimento di Ingegneria dell'Informazione, Università degli Studi di Brescia, Via Branze 38, 25123 Brescia, Italy
}

\section{A R T I C L E I N F O}

\section{Article history:}

Available online 22 January 2011

\section{Keywords:}

Photonic crystals

Bilayer

Coupled membranes

Optical filter

\begin{abstract}
A B S T R A C T
We report on the fabrication of an ultra-compact optical filter based on photonic crystal free-standing membranes in bi-layer configuration. The basic heterostructure consists of two 376 nm-thick GaAs-membranes sandwiched between air on a GaAs substrate. The air gap between the two membranes is $520 \mathrm{~nm}$ thick. The normal-incidence reflectance measurements and the numerical simulation of reflection spectra show a high sensitivity to the holes diameter.
\end{abstract}

(C) 2011 Elsevier B.V. All rights reserved.

\section{Introduction}

One of the most promising applications of photonic crystal technology is in the field of integrated optics and telecommunications [1-3], where it may be useful in the production of compact optical filters, interconnections, wavelength-division multiplexers and other devices.

In the last years a large number of investigations has shown the performance enhancement induced by a new class of optical sensing devices based on photonic crystals (PhCs) free-standing membranes in both single and bi-layer configurations.

Free-standing photonic crystal membranes are very interesting devices as they allow to achieve a good light confinement in the vertical direction due to the high index contrast. This property also makes them attractive for sensors because of the high sensitivity to the variation of filling material refractive index or to external force application.

So far, sensitive devices have been fabricated on a single $\mathrm{PhC}$ slab [4] which has been demonstrated to be the ideal substrate for large scale integration of optical circuits and devices. Huang et al. [5] have introduced a novel sensor scheme combining nano-photonics and nano-fluidics on a single platform through

\footnotetext{
* Corresponding authors.

E-mail addresses: tiziana.stomeo@unisalento.it (T. Stomeo), grande@deemail. poliba.it (M. Grande).

1 These authors equally contributed to the work.

2 Present address: IBM Research GmbH, Saümerstrasse 4, 8803 Rüschlikon, Switzerland.
}

the use of a single free-standing PhC. Suh et al. [6] have described a device based on two parallel photonic crystal slabs and have theoretically demonstrated that a strong variation of the transmissivity of light through such structures can be accomplished with only a nanoscale variation of the spacing between the slabs. This approach relies on the existence of guided resonances in the slabs that allow very efficient coupling with the external incoming light beam $[7,8]$. Coupled bilayer PhC membranes have also been investigated theoretically, leading to both a highly sensitive optical stress sensor [9] and a novel approach for the optomechanical control of light and matter [10]. The fabrication and the demonstration of a large optomechanical coupling in a double slab photonic crystal, suitable as a MEMS actuator, are reported in Ref. [11].

The fabrication of these PhCs is typically carried out using electron-beam lithography and dry etching, and the precise fabrication of the 2D structure is always a critical issue for achievement of low-loss and high-quality components. Well-controlled pattern features are necessary in order to get a $\mathrm{PhC}$ device exhibiting the desired functions. Several fabrication errors must be taken into account as they might cause deviation from perfect photonic-crystal structures realization, such as the deviation of the periodicity due to the hole misplacement, hole size non-uniformity caused by errors in the e-beam writing, proximity-effect-induced non-uniformity in the e-beam lithography, or systematic hole size change due to imperfect sidewall features formed during the dry etching process. The variation of the hole size due to fabrication errors could have a significant impact on the photonic bandgap.

In this work, special attention is given to the electron-beam lithography (EBL) process for making the PhC patterns with well-controlled size, which allows us to successfully fabricate an 
ultra-compact optical filter based on a coupled bilayer PhC. This configuration allowed us to couple and collect light in the vertical direction very efficiently without implementing input and output waveguides.

\section{Experimental}

The implemented optical filter consists of two coupled photonic crystal GaAs-membranes surrounded by air that operates in 1200$1300 \mathrm{~nm}$ spectral window. The basic heterostructure consists of two $376 \mathrm{~nm}$-thick GaAs-membranes sandwiched between air on a GaAs substrate. The air gap between the two membranes is $520 \mathrm{~nm}$ thick. Each membrane consists of a periodic array of air holes into the high-index guiding layer. A square lattice configuration is adopted for the 2D photonic crystal. The sketch of the fabricated device is shown in Fig. 1.

We used a heterostructure with multiple etch-stop layers consisting (from top of the wafer to bottom) of a $376 \mathrm{~nm}$ GaAsmembrane layer, a $520 \mathrm{~nm} \mathrm{Al}_{0.7} \mathrm{Ga}_{0.3}$ As etch-stop layer, a $376 \mathrm{~nm}$ GaAs-membrane layer and a $2000 \mathrm{~nm} \mathrm{Al}_{0.7} \mathrm{Ga}_{0.3} \mathrm{As}$ etch-stop layer on a GaAs substrate. The heterostructure was grown by molecular beam epitaxy (MBE). Both the etch-stop layers are sacrificial and they are removed to realize the two coupled membranes. A $400 \mathrm{~nm}$ hydrogen silsesquioxane (HSQ) resist layer was deposited on the sample as hard mask for high-aspect-ratio etching of photonic crystal patterns [12]. The 2D PhC pattern was written using a Raith150 e-beam lithography system operating at $30 \mathrm{kV}$. A thickness of $400 \mathrm{~nm}$ of ZEP520-A (from Zeon Chemicals L.P.) resist was chosen to ensure sufficient durability as a mask for the transfer of the pattern into the underlying spin-on glass hard mask and, at the same time, to ensure good resolution of e-beam writing. The key issue in the fabrication process is to achieve PhC devices with well-controlled geometrical features size. Indeed, the actual size of the patterned feature is drastically influenced by the electron dose. A preliminary test exposure, referred to as a dose test, has been performed, in which the same pattern has been exposed at different doses and the optimum dose has been determined through inspection in a scanning electron microscope (SEM) after the development process. A proximity error correction (PEC) was also applied to accomplish this target. Moreover, in order to achieve smooth circular holes with fast exposures, our electron beam lithography (EBL) system was used in "circular mode": in this way, every circular hole is exposed by the deflection of the beam along concentric circles. Different high-quality $\mathrm{PhC}$ patterns with well-controlled sizes were achieved after the resist development. The patterns defined in the ZEP resist layer were then transferred into the spin-on glass hard mask using an inductive coupled plasma (ICP) etching (AOE-STS) in fluorine chemistry until the

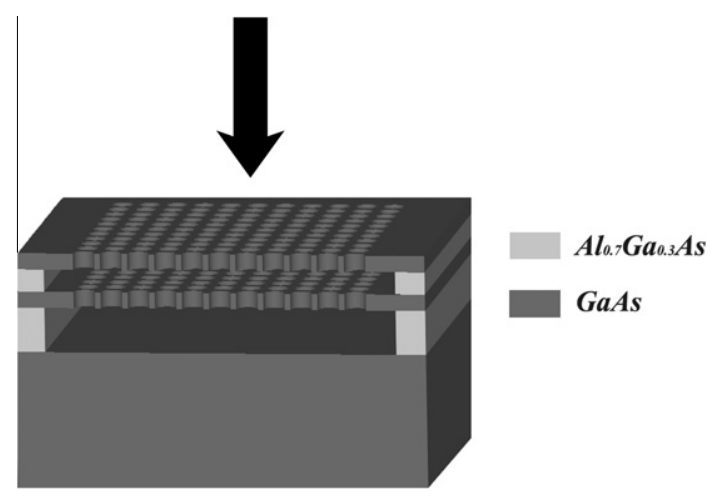

Fig. 1. Sketch of the coupled bilayer photonic crystal.
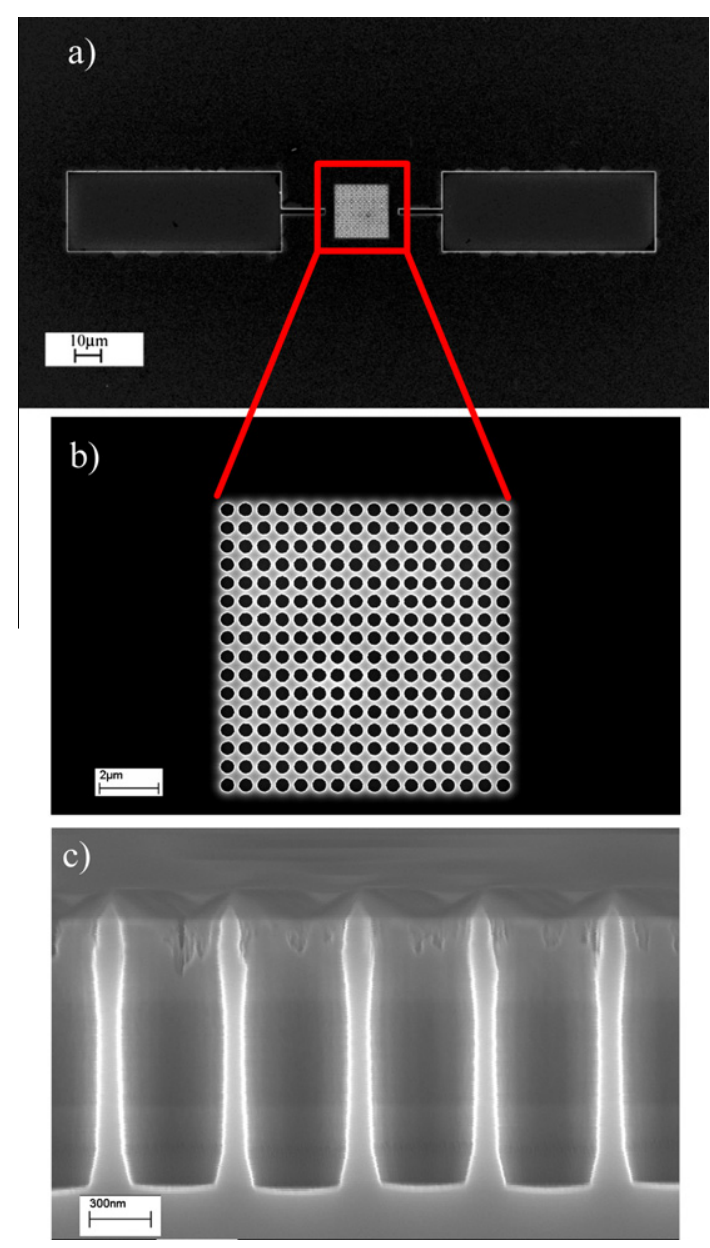

Fig. 2. SEM pictures of the fabricated bilayer PhC free standing membranes: (a) the complete device layout; (b) detail of the 2D PhC pattern (464 nm hole diameter and $627 \mathrm{~nm}$ period); (c) cross-section of the 2D PhC pattern after ICP process where the hole depth is more than $1300 \mathrm{~nm}$ (the upper edge is damaged due to the cleaving process).

GaAs surface is reached. In a following etching step, the holes, defining the square lattice configuration, were deep-etched (more than $1300 \mathrm{~nm}$ ) through the two GaAs waveguide layers and into the sacrificial layer by $\mathrm{SiCl}_{4}$ ICP etching. The final step was the complete removal of the underlying sacrificial $\mathrm{Al}_{0.7} \mathrm{Ga}_{0.3}$ As layers to realize the two PhC membranes. This target was achieved by using a $\mathrm{HF}: \mathrm{H}_{2} \mathrm{O}(1: 10)$ wet etching at room temperature [13] through the photonic crystal holes and the trenches around the periodic pattern. SEM pictures of the fabricated bilayer PhC devices are shown in Fig. 2. The regular square pattern of the PhC slab can be recognized in the middle of Fig. 2a; the rectangular areas on the left and the right sides are the large trenches needed to remove the sacrificial layers by using wet etching. High-quality $\mathrm{PhC}$ patterns (464 nm hole diameter and $627 \mathrm{~nm}$ period) with well-controlled sizes were achieved after the pattern was transferred into the GaAs layers (Fig. 2b). At the same time deep-etched holes and vertical sidewalls were also obtained (Fig. 2c).

Fig. 3a and b show two SEM images (acquired with two different angles) of the realized free-standing bilayer structure where the dark regions correspond to the empty regions, confirming the membranes release.

\section{Results and discussion}

In order to investigate the optical properties of the fabricated free-standing bilayer PhC structure, we performed reflectance 

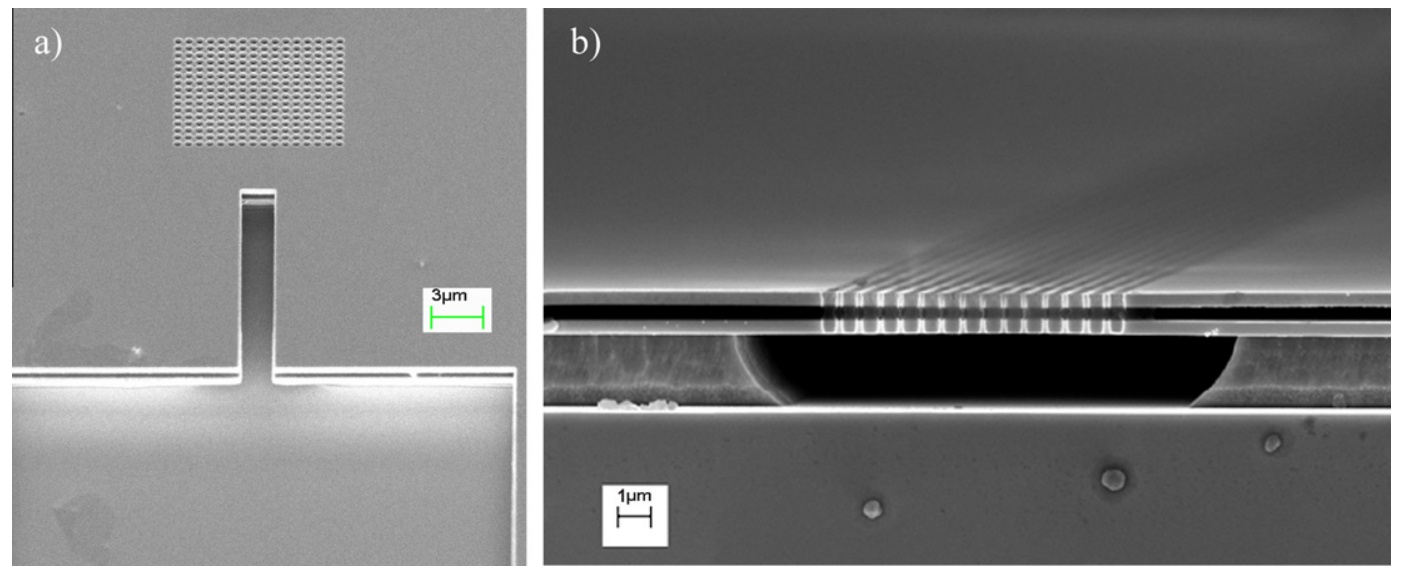

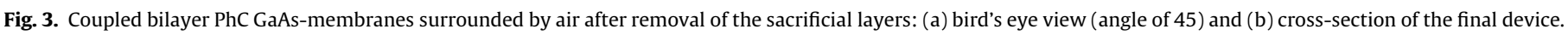

measurements by using a $\mu$-reflectance set-up. A light probe beam (from a tungsten broad band lamp) was focused onto the sample by a microscope objective. The reflected beam was then collected by the same microscope objective and measured by a monochromator coupled to an N-cooled InGaAs OMA (Optical Multichannel Analyzer). In Fig. 4, we plot the measured reflection spectra for two different hole diameters. There are two distinct reflectivity dips (i.e., the peak of transmissivity) in the spectrum at $1200 \mathrm{~nm}$ and $1185 \mathrm{~nm}$, which clearly indicate a resonant behaviour for the $459 \mathrm{~nm}$ and $464 \mathrm{~nm}$ hole diameter devices, respectively. The measured resonance wavelengths have been compared with the calculated values which have been carried out by means of a 3D finite-difference time-domain (FDTD) code, as described in a previous work [14]. The normal-incidence reflectance measurements and the numerical simulations of reflection spectra show a high sensitivity to the holes diameter. This confirms that the coupled bilayer photonic crystal behaves as a Fabry-Perot cavity whose reflectivity and transmissivity depend on the geometrical parameters (i.e., air gap between the two membranes and holes diameter).

It is worth to pointing out that the non-zero cavity reflection can be related to the fabrication tolerances with respect to the nominal geometrical parameters. In particular the SEM inspection reveals a slight bending of the two free-standing $\mathrm{PhC}$ membranes as shown in Fig. 3b. At the same time, the roughness of the PhC

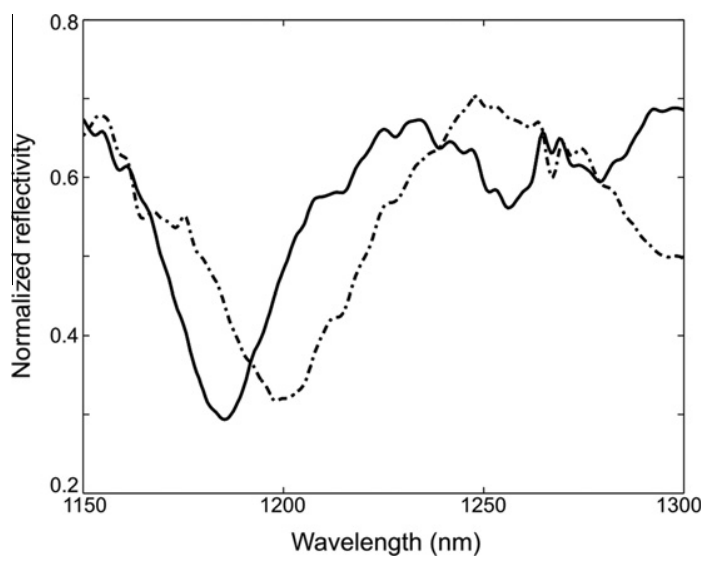

Fig. 4. Measured reflection spectra of the two coupled GaAs-membranes surrounded by air for two different values of holes diameter: $459 \mathrm{~nm}$ (dashed-dotted line) and $464 \mathrm{~nm}$ (continuous line), respectively. sidewalls can cause scattering and lower reflectivity of the PhC mirrors $[15,16]$.

\section{Conclusion}

In conclusion, we have demonstrated a coupled bilayer GaAs photonic crystal optical filter and highlighted that the optical tunability, performance and properties of devices are quite sensitive to the fabrication accuracy and tolerances. Along this line, the same technology is being applied for fabricating such an optical filter by filling the air-gap with a polymeric matrix, thus improving the mechanical strength of the device and paving the way for a new class of optical stress/pressure sensing devices based on two coupled $\mathrm{PhC}$ membranes operating in both visible and infrared spectral range. 2D bilayer PhCs employed in this study are also promising for fabricating new coupled optomechanical systems due to the strong light confinement and the possibility to provide ultrahigh-Q performance. In particular 2D PhCs slab geometry could also show potential for the fabrication of micro-electro-mechanical systems (MEMS), Micro-opto-electro mechanical systems (MOEMS), tunable OCT filter and high sensitivity fluorescence detection-based biosensors.

\section{Acknowledgements}

The authors gratefully thank Dr. Antonio Qualtieri and the expert technical help of Gianmichele Epifani, Iolena Tarantini, Adriana Campa e Gianvito De Iaco. This work was supported by MIUR-PRIN2005, in the framework of the national research project "Photonic Band Gap Nanosensors". This work was supported by Firb - Hub di ricerca italo-giapponese sulle nanotecnologie.

\section{References}

[1] T.F. Krauss et al., Prog. Quant. Electron. 23 (1999) 51

[2] T. Stomeo et al., Opt. Lett. 33 (2008) 884.

[3] M. Grande et al., Opt. Lett. 34 (2009) 3294.

[4] S.G. Johnson, S. Fan, P.R. Villeneuve, J.D. Joannopoulos, L.A. Kolodzjeski, Phys. Rev. B 62 (2000) 8212.

[5] M. Huang, A.A. Yanik, T. Chang, H. Altug, Opt. Express 17 (26) (2009) 24224.

[6] W. Suh, M.F. Yanik, O. Solgaard, S. Fan, Appl. Phys. Lett. 82 (2003) 1999.

[7] M. Kanskar, P. Paddon, V. Pacradouni, R. Morin, A. Busch, J.F. Young, S.R. Johnson, J. Mackenzie, T. Tiedje, Appl. Phys. Lett. 70 (1997) 1438.

[8] S. Fan, J.D. Joannopoulos, Phys. Rev. B 65 (2002) 235112.

[9] T. Lu, P. Lee, Opt. Express 17 (3) (2009) 1518

[10] M. Notomi, H. Taniyama, S. Mitsugi, E. Kuramochi, Phys. Rev. Lett. 97 (2006) 023903. 
[11] Y. Rho, T. Tanabe, A. Shinya, H. Taniyama, E. Kuramochi, S. Matsuo, T. Sato, M. Notomi, Phys. Rev. B 81 (2010) 121101(R).

[12] L. O'Faolain, M.V. Kotlyar, N. Tripathi, R. Wilson, T.F. Krauss, J. Vac. Sci. Technol. B 24 (2006) 336.

[13] T. Stomeo, V. Errico, A. Salhi, A. Passaseo, R. Cingolani, A. D’Orazio, V. Marrocco, M. De Vittorio, Microelectron. Eng. 83 (2006) 1823.
14] T. Stomeo, M. Grande, G. Rainò, A. Passaseo, A. D’Orazio, R. Cingolani, A. Locatelli, D. Modotto, C. De Angelis, M. De Vittorio, Opt. Lett. 35 (3) (2010) 411.

[15] H. Shin, Y. Ju, H. Song, D. Song, Il-Y. Han, J. Ser, H. Ryu, Y. Lee, Appl. Phys. Lett 72 (1998) 2205.

[16] B.E.A. Saleh, M.C. Teich, Fundamentals of Photonics, Wiley, 1991. 